\title{
Design and Development of Modified mRNA Encoding Core Antigen of Hepatitis C Virus: a Possible Application in Vaccine Production
}

\author{
Zarin Sharifnia ${ }^{1,2,3}$, Mojgan Bandehpour ${ }^{3,4}$, Bahram \\ Kazemi ${ }^{4}$ and Nosratollah Zarghami ${ }^{1,2,5^{*}}$
}

\begin{abstract}
${ }^{1}$ Drug Applied Research Center, Tabriz University of Medical Sciences, Tabriz, Iran; ${ }^{2}$ Department of Medical Biotechnology, Faculty of Advanced Medical Sciences, Tabriz University of Medical Sciences, Tabriz, Iran; ${ }^{3}$ Cellular and Molecular Biology Research Center, Shahid Beheshti University of Medical Sciences, Tehran, Iran; ${ }^{4}$ Department of Biotechnology, School of Advanced Technologies in Medicine, Shahid Beheshti University of Medical Sciences, Tehran, Iran; ${ }^{5}$ Department of Clinical Biochemistry and Laboratories Medicine, Faculty of Medicine, Tabriz University of Medical Sciences, Tabriz, Iran
\end{abstract}

\begin{abstract}
Background: Hepatitis $\mathrm{C}$ virus ( $\mathrm{HCV})$ is a blood-borne pathogen, resulting in liver cirrhosis and liver cancer. Despite of many efforts in development of treatments for HCV, no vaccine has been licensed yet. The purpose of this study was to design and prepare a specific mRNA, without $5^{\prime}$ cap and poly (A) tail transcribed in vitro capable of coding core protein and also to determine its functionality. Methods: Candidate mRNA was prepared by in vitro transcription of the designed construct consisting of $5^{\prime}$ and $3^{\prime}$ untranslated regions of heat shock proteins 70 (hsp70) mRNA, T7 promoter, internal ribosome entry site (IRES) sequences of elF4G related to human dendritic cells (DCs), and the Core gene of HCV. To design the modified mRNA, the $5^{\prime}$ cap and poly (A) tail structures were not considered. DCs were transfected by in vitro-transcribed messenger RNA (IVT-mRNA) and the expressions of green fluorescent protein (GFP), and Core genes were determined by microscopic examination and Western blotting assay. Results: Cell transfection results showed that despite the absence of 5' cap and poly (A) tail, the structure of the mRNA was stable. Moreover, the successful expressions of GFP and Core genes were achieved. Conclusion: Our findings indicated the effectiveness of a designed IVT-mRNA harboring the Core gene of HCV in transfecting and expressing the antigens in DCs. Considering the simple and efficient protocol for the preparation of this IVT-mRNA and its effectiveness in expressing the gene that it carries, this IVT-mRNA could be suitable for development of an RNA vaccine against HCV. DOI: 10.29252/ibj.23.1.57
\end{abstract}

Keywords: Hepatitis C, Messenger, RNA, Vaccines

Corresponding Author: Nosratollah Zarghami

Drug Applied Research Center, Tabriz University of Medical Sciences, Tabriz, Iran; Tel.: (+98-41) 33364666; E-mail: zarghami@tbzmed.ac.ir

\section{INTRODUCTION}

$\mathrm{H}$ epatitis C virus (HCV) is a blood-borne pathogen and an enveloped, single-stranded, and positive-sense RNA virus belonging to Flaviviridae family ${ }^{[1,2]}$. It is estimated that about $2 \%$ $3 \%$ of the world's population is infected with $\mathrm{HCV}^{[3]}$, and most of the acute hepatitis $\mathrm{C}$ infections become chronic. If left untreated, the chronic disease can lead to cirrhosis and hepatocellular carcinoma in a number of patients. At the moment, chronic hepatitis $\mathrm{C}$ infection can be treated by antiviral therapy ${ }^{[1,4-6]}$. In recent years, direct-acting antivirals (DAAs) regimens has been introduced due to its high efficacy rate 
(>95\%) against all genotypes of the HCV. However, there are major barriers to widespread use of DAAs regimens, including geographical factors with limited availability of new compounds, virus factors like $\mathrm{HCV}$ genotype, host factors such as prolonged liver damage even after treatment, inability to prevent re-infection, and the high cost of DAAs drugs ${ }^{[7-9]}$. Although antiviral agents show a great efficacy in $\mathrm{HCV}$ treatment, it seems that the global burden of liver disease does not decrease without the combination of effective antiviral treatments, appropriate screening techniques, and preventive vaccine development. Currently, no vaccine has been licensed or administered for HCV, but trials are under way ${ }^{[10]}$.

A wide variety of vaccines have been designed so far such as protein-based vaccines like recombinant glycoproteinE1/glycoprotein E2 adjuvanted with MF59 and recombinant $\mathrm{HCV}$ core antigen formulated with the T-cell adjuvant IMX, inactivated cell culturederived $\mathrm{HCV}$ virions, virus-like particles presenting $\mathrm{HCV}$ envelope proteins, viral-vectored vaccines expressing conserved HCV NS3-NS5B non-structural proteins (Ad6-Nsmut/AdCh3-Nsmut and MVANSmut/AdCh3-NSmut vaccines), as well as DNA plasmid encoding the HCV proteins $3 / 4 \mathrm{a}^{[11,12]}$.

mRNA is a transient carrier of genetic information that is naturally metabolized and does not integrate to the genome. The synthetic mRNA can be easily designed to express any antigen with high efficiency, and it can also act as an adjuvant in vaccine formulations. Since mRNA, in contrast to DNA, is located and acts in the cytoplasm, it easily can transfect all cell types, especially hard-to-transfect cells. Another advantages of using mRNA in a treatment approach are its cost-effective manufacturing and its easy scalability. As mRNA transcription is possible in vitro, production of high dose vaccines in a short time is achievable, which allows the rapid production of a vaccine for a new antigen during pandemics ${ }^{[13-17]}$. Typically, RNA vaccines consist of a mRNA synthesized via an in vitro transcription using a bacteriophage RNA polymerase and a DNA template encoding the antigen of interest ${ }^{[18]}$.

Vaccine development using mRNA approaches have advantages over other vaccine methods ${ }^{[19]}$. mRNA vaccines is promising to prevent and treat a wide range of diseases such as influenza, rabies, or cancers ${ }^{[18,20]}$. However, no $\mathrm{HCV}$ vaccine has been designed using this methodology.

Recently, studies have used in vitro transcribedmessenger RNA (IVT-mRNA), as a gene carrier, in subunit vaccines to supply therapeutic proteins. in vitro protein expression of brome mosaic virus and poliovirus RNA has been confirmed ${ }^{[21,22]}$, and based on this fact, several vaccines have been designed against viral infections ${ }^{[23]}$. Studies have indicated the effectiveness of dendritic cells (DCs) transfected with antigen-encoding mRNA genes in cancer immunotherapy ${ }^{[24,25]}$. Although mRNA approaches is being used widely in several clinical trials in cancer therapy ${ }^{[26]}$ IVT-mRNA is supposed to be too immunogenic and labile for genetic introduction of proteins. To overcome these challenges, several strategies have been developed, that include the replacement of modified nucleotides with unmodified counterpart, codon optimization of an IVT-mRNA sequence for enhance protein production, using untranslated regions (UTRs) in the structure of mRNA, and the use of safe delivery tools ${ }^{[27]}$. In order to develop the cheaper and more flexible technology of IVT-mRNA production, focusing on the above mentioned strategies may be useful.

Our aim here was to design and prepare a synthetic mRNA that encodes the most conserved protein of $\mathrm{HCV}$, core protein and also to determine its ability to encode the protein in DCs. In the present study, we have made a sequence consisted of 5' and 3' UTRs of heat shock proteins 70 (hsp70) mRNA, T7 promoter, internal ribosome entry site (IRES) fragments that enable eIF4G capture for the initiation of translation to express HCV Core gene and Core/signal peptide (SP) in human DCs.

\section{MATERIALS AND METHODS}

\section{Plasmid constructs}

To prepare a pGE-Core construct, Core protein consisting of 1-191 aa (573 bp) of HCV-1a strain Tehran 12 (GenBank: AF512996.1, 2002) was ligated into the pGE-30446-HCE vector using Pst I restriction enzyme. This plasmid was constructed under the control of $\mathrm{T} 7$ bacteriophage promoter with 5' UTR, ORF, IRES, and 3' UTR. The 5' UTR and 3' UTR were from human hsp70 along with IRES sequences of human DCs This synthetic construct was provided commercially( Generay, China (Fig. 1A).

In order to secrete the Core protein from the DCs, the sequence of the SP of the CD86 marker (accession number: P42081) on the surface of the DCs was added to the 5' region of the Core gene using overlap extension PCR method (Fig. 2A). Four pairs of primers used for the amplification of the fragment are listed in Table 1. The amplified PCR product was digested by PstI restriction enzymes and was subcloned into the pGE-30446-HCE synthetic plasmid. To construct a reporter gene, the sequence of the green fluorescent protein (GFP) from pEGFP-N1 vector was amplified 


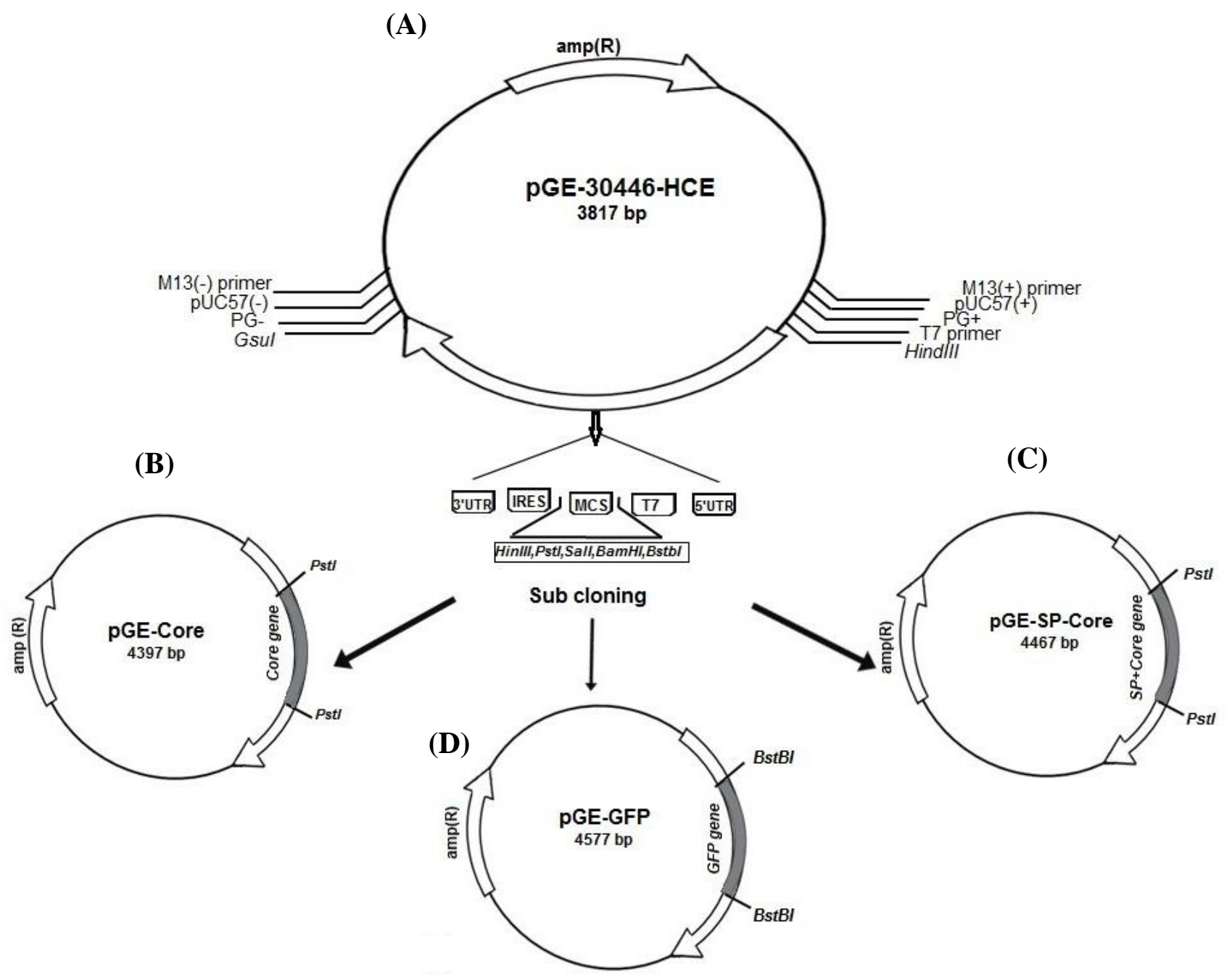

Fig. 1. Schematic representation of plasmid construction procedure. (A) The designed pGE-30446-HCE plasmid containing synthesis gene with the following characteristics: gene name, 30446- HCE; gene length, 915 bp; orientation, M13forward-geneM13reverse; cloning site, Ec072I; vector, pGE; (B) pGE-30446-HCE plasmid after subcloning of HCV Core protein-coding sequence in PstI restriction site; (C) pGE-30446-HCE plasmid after subcloning of HCV SP-Core-coding sequence in PstI restriction site; (D) pGE-30446-HCE plasmid after subcloning of GFP-coding sequence in BstBI restriction site.

(Table 1) and ligated into pGE-30446-HCE vector using the BstBI restriction enzyme. The accuracy of the constructs was verified by sequencing.

\section{Preparation of in vitro transcription mRNA}

IVT-mRNAs were synthesized from pGE-Core, pGE-SP-Core, and pGE-GFP vectors (Fig. 1B, 1C, and 1D) after the linearization of the constructs with HindIII and GsuI restriction enzymes (MBI Fermentas, St Leon-Rot, Germany). The IVT-mRNAs synthesis was performed using RiboMAX ${ }^{\mathrm{TM}}$ Large-Scale RNA Production Systems-SP6 and T7 Kit (Promega Corporation, Madison, USA) according to the manufacturer's instruction. DNA templates were purified using a PCR purification kit and used for in vitro transcription. Transcription of 5-10 $\mu \mathrm{g}$ of the DNA templates was carried out in a final 20-200 $\mu 1$ reaction mixture at $37{ }^{\circ} \mathrm{C}$ for 4 hours in order to generate uncapped IVT-mRNA. To remove the template DNA, $1 \mu$ of RQ1 RNase-Free DNase (Catalog Numbers: M6101) was added to the IVT reaction mixture and incubated at $37^{\circ} \mathrm{C}$ for $15 \mathrm{~min}$. Purification of mRNA was performed after DNaseI digestion by Total RNA Purification Kit (JenaBioscience, Germany), according to the instructions provided by manufacturer. The purity and quality of the RNAs were determined using spectrophotometric analysis and run on the agarose gel (1.1\%). The mRNAs were stored in the RNase-free water at $-80{ }^{\circ} \mathrm{C}$. 
Table 1. Gene specific-primers used to amplify the core, SP-core, and GFP genes.

\begin{tabular}{lll}
\hline Gene & Forward Primer $\left(\mathbf{5}^{\prime} \mathbf{-} \mathbf{3}^{\prime} \mathbf{)}\right.$ & \multicolumn{1}{c}{ Reverse Primer $\left(\mathbf{5}^{\prime} \mathbf{-} \mathbf{3}^{\prime} \mathbf{)}\right.$} \\
\hline Core & ATATATCTGCAGATGAGCACGAATCCTAA & \\
& F1:CTGCTGAGCGCCATGAGCACGAATCCTAA & ATATATCTGCAGTCAGGCTGACGCGGGCACAGT \\
& F2:GAGCAACATCCTGTTCGTGATGCCTTCCTGCTG & CA \\
SP-Core & F3:AGTGCACCATGGGCCTGAGCAACATCCTGTTCAC & ATATATCTGCAGTCAGGCTGACGCGGGCACAGT \\
& GCG & CA \\
& F4:ATATATATCTGCAGATGGACCCCCAGTGCACCAT & \\
& GG & ATATACTTCGAAACAGCTCGTCCATGCC \\
\hline
\end{tabular}

Start and stop codons are shown in bold.

\section{Computational analysis of mRNA secondary structure}

The secondary structure of designed mRNA was analyzed using the RNA structure software package (http://rna.urmc.rochester.edu/RNAstructureWeb). The free energy of the mRNA was calculated using a dynamic planning algorithm to predict the most stable molecule. RNA structure software not only displays the thermodynamic properties of the sequence but also includes an interactive graphical representation of the predicted secondary structure ${ }^{[28]}$.

\section{Generation of monocyte-derived DCs}

Human monocyte-derived DCs were generated as described elsewhere ${ }^{[29]}$. Briefly, fresh human peripheral blood mononuclear cells were isolated from healthy donors by density gradient centrifugation over Ficoll-Hypaque. The blood cells were cultured for $2 \mathrm{~h}$. Adherent monocytes were washed with RPMI-1640 medium and cultured for seven days in RPMI-1640 medium supplemented with 10\% FBS, L-glutamine (2mM), penicillin (100 IU), streptomycin $(100 \mu \mathrm{g} / \mathrm{mL})$, IL-4 (25 ng/mL), and recombinant granulocytemacrophage colony-stimulating factor $(50 \mathrm{ng} / \mathrm{mL})$ in a $\mathrm{CO}_{2}$ incubator at $37{ }^{\circ} \mathrm{C}$. After seven days, immature monocyte-derived DCs (imoDCs) were ready for treatment.

\section{Transfection of imoDCs with IVT-mRNA}

moDCs were transfected with $1.5 \mu \mathrm{g}$ of IVT-mRNA using Lipofectamine 2000 (Invitrogen, USA), an RNA transfection reagent, according to the manufacturer's instructions. The modification of IVT-mRNA with lipofectamine was performed as per manufacturer's protocol. The moDCs were then incubated in a $\mathrm{CO}_{2}$ incubator at $37{ }^{\circ} \mathrm{C}$ for $18-48 \mathrm{~h}$ prior to testing for transgene expression. The expressions of GFP and $\mathrm{HCV}$ core protein were measured 24-48 $\mathrm{h}$ after transfection by fluorescence microscopy (Nikon Eclipse TE2000-U, USA) and Western blotting assay.

\section{Detection of Core and GFP protein expressions in DCs by Western blotting}

After the transfection of imoDCs with IVT Core and GFP mRNA, the transfected and control cells were sonicated in a protein lysis buffer containing $50 \mathrm{mM}$ of Tris, $50 \%$ glycerol, $0.1 \%$ Triton $\mathrm{X}-100,1 \mathrm{mM}$ of antiprotease (phenylmethylsulfonylfluoride). The acetoneprecipitated proteins were loaded on $10 \%$ SDSpolyacrylamide gel and subsequent Western blot analysis was carried out using positive anti-HCV antibody serum specimens, which had been diluted $1: 100$ in $1 \times$ TBS buffer. After washing, the membrane was incubated with secondary peroxidase-conjugated anti-human IgG antibody for $2 \mathrm{~h}$, followed by washing with TBST buffer. The bands were visualized using the chromogenic substrate 3,3'-diaminobenzidine (Sigma, USA). The untransfected cells were included as negative controls. The expression of GFP protein in the transfected cells was analyzed in the same way using 1:1500 diluted anti-GFP- antibody (Abcam, UK) and 1:5000 diluted anti-rabbit antibody conjugated with Alkaline phosphatase as the first and second antibodies, respectively. The protein was visualized by adding BCIP/NBT solution as a detection substrate.

\section{RESULTS}

\section{Stable secondary structure of Core mRNA}

The structure of synthesized fragment containing 5' UTR, T7 promoter, multiple cloning site, IRES, and 3' UTR (915 bp) with and without Core (1497 bp) and SP-Core (1565 bp) proteins coding sequences was predicted using RNA structure program. RNA structure utilizes the nearest neighbor parameters and thermodynamic parameters to predict the lowest free energy structure of $\mathrm{RNA}^{[30]}$. In general, among a series of same-sized RNAs, lower thermodynamic energy represents a more stable structure. Figure 3 shows the predicted RNA structures with the lowest free energy 


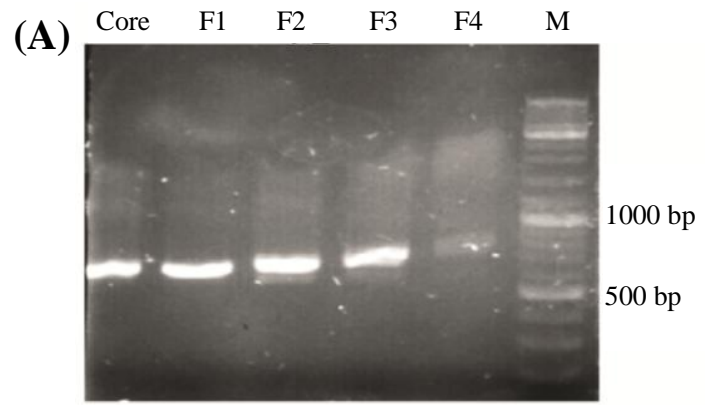

(C)

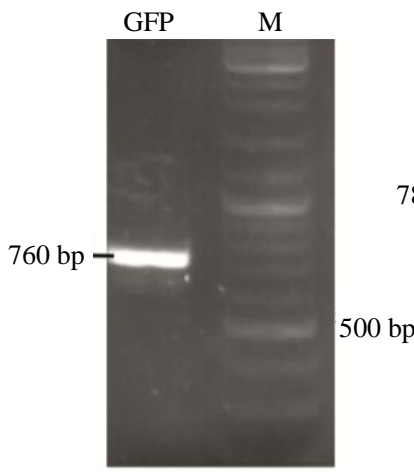

(D)

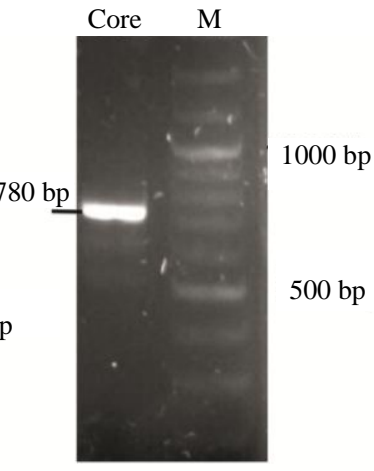

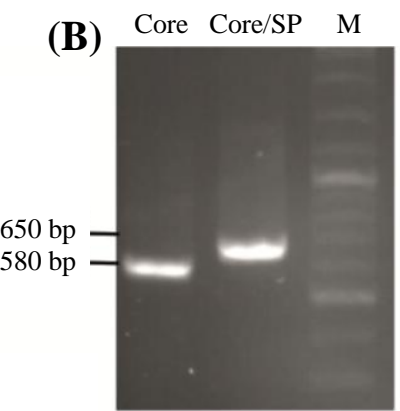

(E)

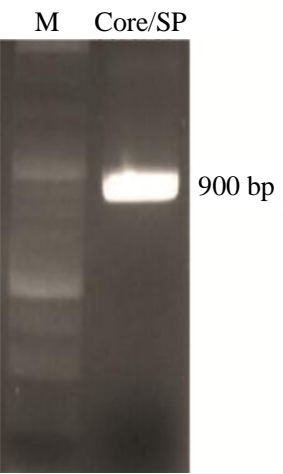

Fig. 2. The result of amplification of coding sequences and splicing by overlap extension-PCR reaction analyzed by gel electrophoresis. (A) PCR products of the Core and SP-Core coding sequences, (B) PCR products related to the step-by-step addition of the SP to the Core-coding sequence using SOE-PCR. F1-F4 indicate the PCR result of using primers F1-F4 (Table 2) to extend core sequence. PCR result of cloning confirmation of (C) GFP, (D) Core, and (E) SP-Core in pGE-30446-HCE using the forward primer of the M13 and the reverse primer of the core gene. M, marker

for three designed RNAs. In the predicted structures of Core and SP-Core, several regions with paired and unpaired nucleotides have been indicated. Singlestranded regions indicate regions with the low probability of pairing. The calculated minimum free energy for the secondary structure of synthesized sequence alone and containing Core and SP-Core coding sequences were $-307.3,-611.3,-647.2 \mathrm{kcal} / \mathrm{mol}$, respectively. We compared the calculated free energy of $\mathrm{HCV}$ synthetic mRNAs with that of native mRNA sequences carried out in other studies with similar lengths of sequences, assuming that the synthesized mRNA in this study had a reasonable stability (Table $2)^{[31,32]}$

\section{Preparation of template plasmid expressing pGE- Core, pGE-SP-Core, and pGE-GFP proteins}

The sequences related to the 5' UTR, T7 promoter, MCS, IRES, and 3' UTRs of the plasmid with the length of $915 \mathrm{bp}$ were synthesized by GeneRay Co. We assumed that adding known 5 ' and $3^{\prime}$ UTRs to the construct will stabilize the IVT mRNA structure and will increase the efficiency of translation. Based on the studies conducted in this area, a structure of the 5' UTR of human hsp70 enhances the translation of mRNA in mammalian cells and is predicted to be valuable in the context of genetic vaccination ${ }^{[33,34]}$. To confirm the subcloning of Core and SP-Core coding sequences in PstI restriction site, PCR test was conducted using specific primers (Fig. 2B). In order to confirm the subcloned GFP sequence by $B s t \mathrm{BI}$ restriction enzyme, the resulting clone was amplified using GFP-specific primers (Fig. 2C). GFP is a widely used reporter protein for monitoring gene expression in vivo and in vitro and for investigating the intracellular pattern of protein localization and trafficking. As GFP is more sensitive than other reporter genes, it requires no special cofactor for detection. Also, the intensity of GFP fluorescence is directly proportional to GFP mRNA abundance in the cells ${ }^{135,36]}$. In the current study, GFP protein was used as a quantitative reporter of gene expression in individual DCs. Clones with the correct orientation of gene were selected with a confirmatory PCR reaction using the forward primer of the M13 and the reverse primer of the Core gene (Fig. $2 \mathrm{D}$ and $2 \mathrm{E}$ ). 
Table 2. Minimal free energies of native mRNA with predicted energies for mRNA synthesized in this study

\begin{tabular}{|c|c|c|c|c|c|}
\hline \multirow{2}{*}{ Gene name } & \multicolumn{2}{|c|}{ Native mRNA } & \multirow{2}{*}{ Gene name } & \multicolumn{2}{|c|}{ Synthetic mRNA } \\
\hline & Length & MFE & & Length & MFE \\
\hline HUMGST (glutathione S-transferase) & 909 & -204 & Synthetic sequence & 915 & -307.3 \\
\hline$T H A R G A A(t R N A)$ & 1471 & -650.1 & $\begin{array}{l}\text { Synthetic sequence (with Core gene) } \\
\text { Synthetic sequence (with SP-Core gene) }\end{array}$ & $\begin{array}{l}1497 \\
1565\end{array}$ & $\begin{array}{l}-611.3 \\
-647.2 \\
\end{array}$ \\
\hline
\end{tabular}

MFE, minimum free energy

\section{In vitro transcription process}

To perform a T7 IVT, prepared plasmids containing DNA sequence with known flanking sequences were digested by HindIII and GsuI restriction enzymes. The lengths of resulting fragments for plasmids containing Core, SP-Core, and GFP coding sequences were 1497, 1565 , and $1660 \mathrm{bp}$, respectively. These fragments were purified, and the quality of the generated DNA was determined using spectrometry, calculating A260/A28 0 ratio, as well as agarose gel (1.5\%) electrophoresis (Fig. 4A). In vitro transcription was performed from DNA templates. RNA concentration was assayed by a spectrophotometric analysis at OD $260 \mathrm{~nm}$. The concentration of mRNA synthesized in a triplicate experiment was between $2-2.4 \mathrm{mg} / \mathrm{mL}$ of RNase-free water. The purity of the mRNA was determined by A260/A280 ratio, which was in the range of 1.6 to 2.0. Given the normal range of 1.8 to 2.1 for the ratio, the quality of synthesized RNAs was acceptable. To check the size and the quality of mRNA, a small sample of synthetic mRNA was loaded on a $1.1 \%$ agarose gel. After electrophoresis, the HCV Core mRNA transcript with a length of about $1.6 \mathrm{~kb}$ was detected on an agarose gel (Fig. 4B).
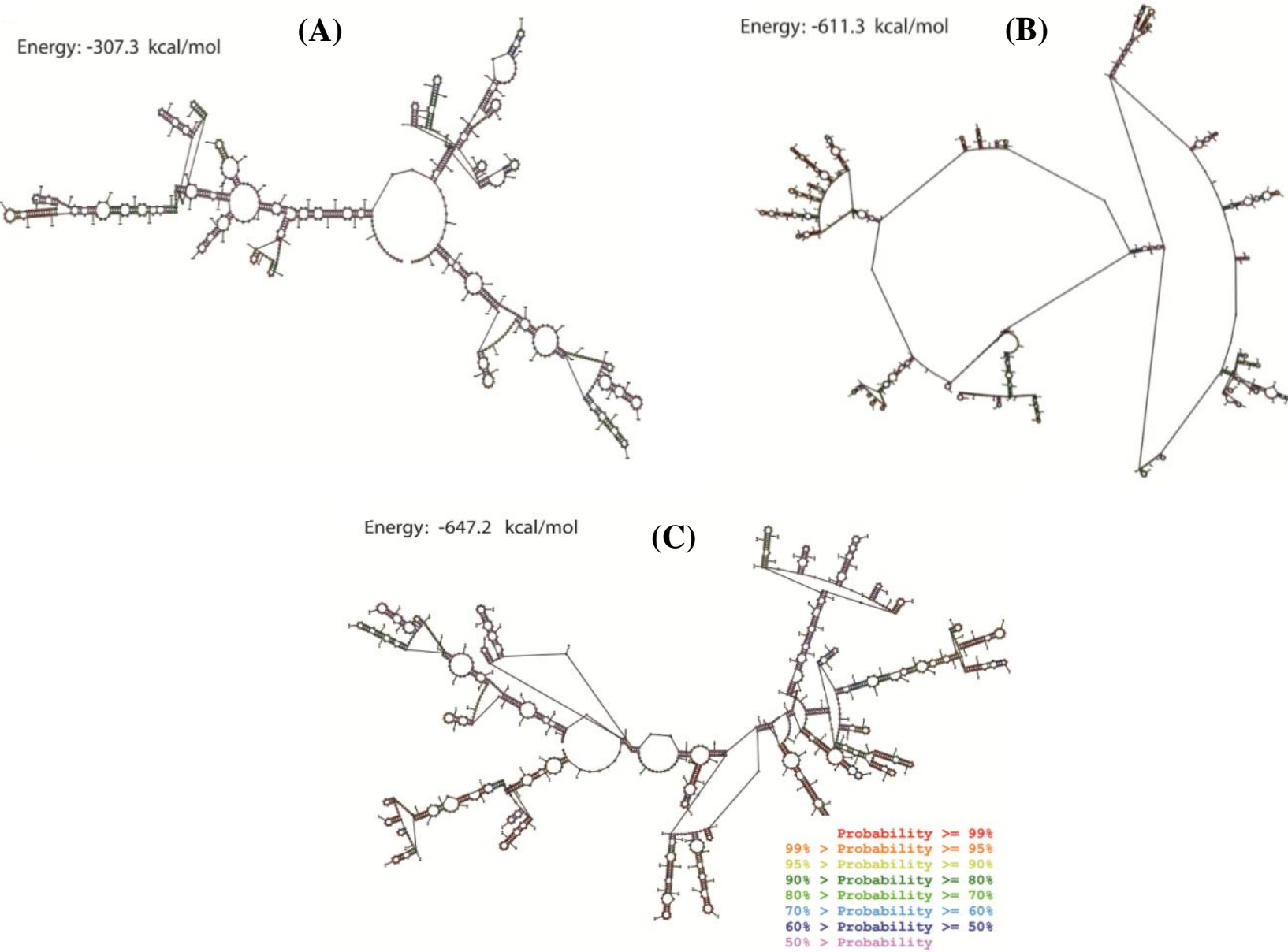

Fig. 3. Predicted probability of nucleotides being paired or single stranded using RNA structure program. Predicted probability of nucleotides of (A) synthesized backbone sequence RNA, (B) Core protein RNA, and (C) SP-Core RNA. Probability lower than 50\% is not colored. The calculated minimum free energies for secondary structure of synthesized sequence alone and containing Core and SP-Core coding sequences were $-307.3,-611.3,-647.2 \mathrm{kcal} / \mathrm{mol}$, respectively. 

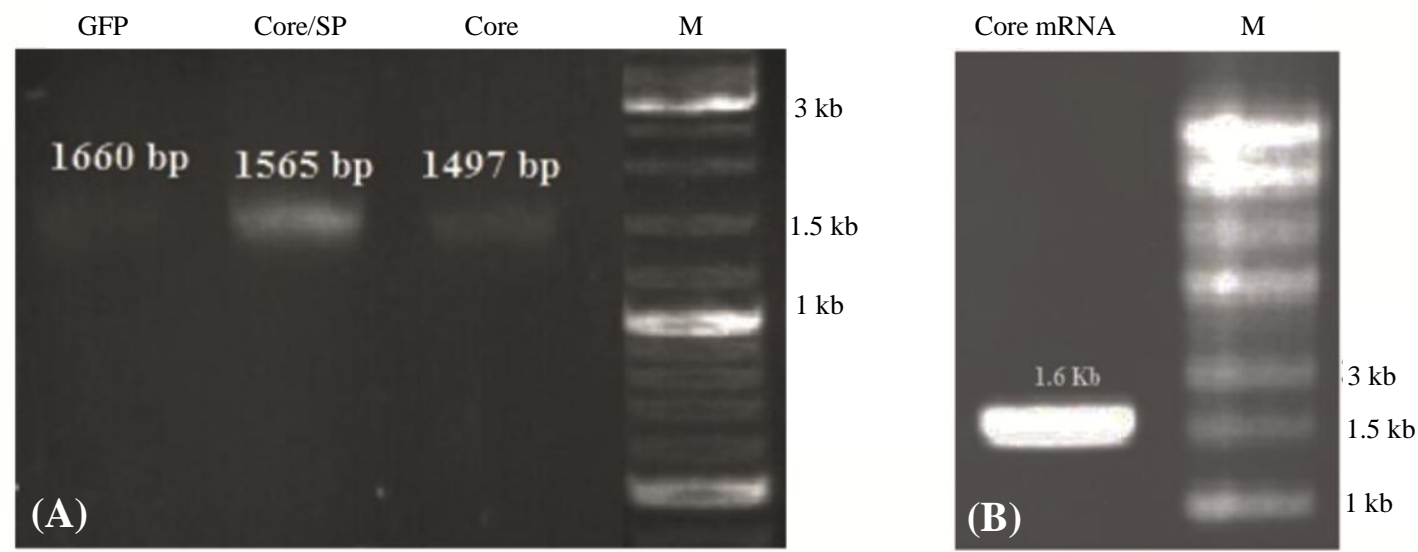

Fig. 4. PCR products of cloned sequences and mRNA transcript. (A) The amplified fragments obtained from the enzymatic digestion of plasmids carrying Core, SP-Core, and GFP genes; (B) The amplified HCV core mRNA transcript (1.6 kb) running on an agarose gel $(1.1 \%)$. M, RNA marker

\section{Effective expression of designed mRNA in moDCs}

The transfection of moDCs using lipofectamine resulted in a relatively good transfection efficiency. The GFP expression in the cells was measured by fluorescence intensity and Western blotting. The moDCs were transfected using $2 \mu \mathrm{g}$ of GFP mRNA along with $2 \mu \mathrm{l}$ of Lipofectamine 2000. After 24-48 h, the GFP expression in the cells was investigated by fluorescence microscopy (Fig. 5). The analyses demonstrated strongly fluorescent moDCs. The Core protein expression was assayed in the moDCs via Western blotting. As illustrated in Figure 6A, Core protein was observed as a multi-band protein with different molecular masses $(37-55 \mathrm{kDa})$ in the cell extracts obtained after the transfection of the moDCs. The observed additional bands, particularly the one with the molecular weight of $44 \mathrm{kDa}$, might correspond to the dimer form of the Core protein. The presence of proteins with other molecular weights suggests that the formation of complex products is possible. Also, the expression of GFP in moDCs was screened by Western blotting after the transfection of its mRNA with lipofectamine. As shown in Figure 6B, a protein with the expected molecular mass $(27 \mathrm{kDa})$ was observed in the cell extracts. The Western blotting on untransfected moDCs, as negative control, has also been carried out (Fig. 6).

\section{DISCUSSION}

In the field of vaccine development, it is necessary to pay attention to its scientific, technological, and economic aspects. Therefore, the challenges that need to be addressed are (A) the lack of efficiency against highly variable and/or persistent pathogens, (B) the development of a cheaper and more flexible technology to use in the absence of resources, and $(\mathrm{C})$ better adaptation for individual or rapid production. Considering the mentioned issues and the characteristics of synthetic mRNA, it seems that synthetic mRNA could be of particular importance in the future of vaccine development, especially for highly variable infectious agents ${ }^{[37,38]}$.

Synthetic mRNA allows creating the optimal immunogenic characteristics of a pathogen by eliminating unnecessary or harmful aspects of a pathogen. Due to its chemical and pharmacokinetic nature, IVT-mRNA may mimic the RNA of viruses; however, it causes an infection in a transient manner ${ }^{[39]}$. To design an effective mRNA-based

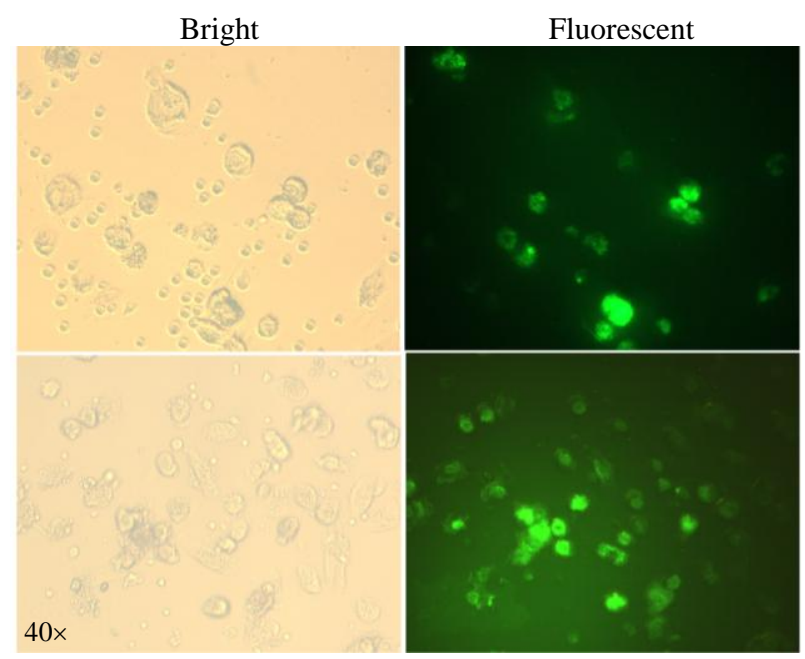

Fig. 5. GFP expression in DCs using fluorescence microscopy. Immature DCs were transfected with IVT-mRNA expressing GFP and were analyzed for GFP protein expression $48 \mathrm{~h}$ after transfection. GFP protein expressed in DCs is indicated in fluorescent microscopy fields (right). 

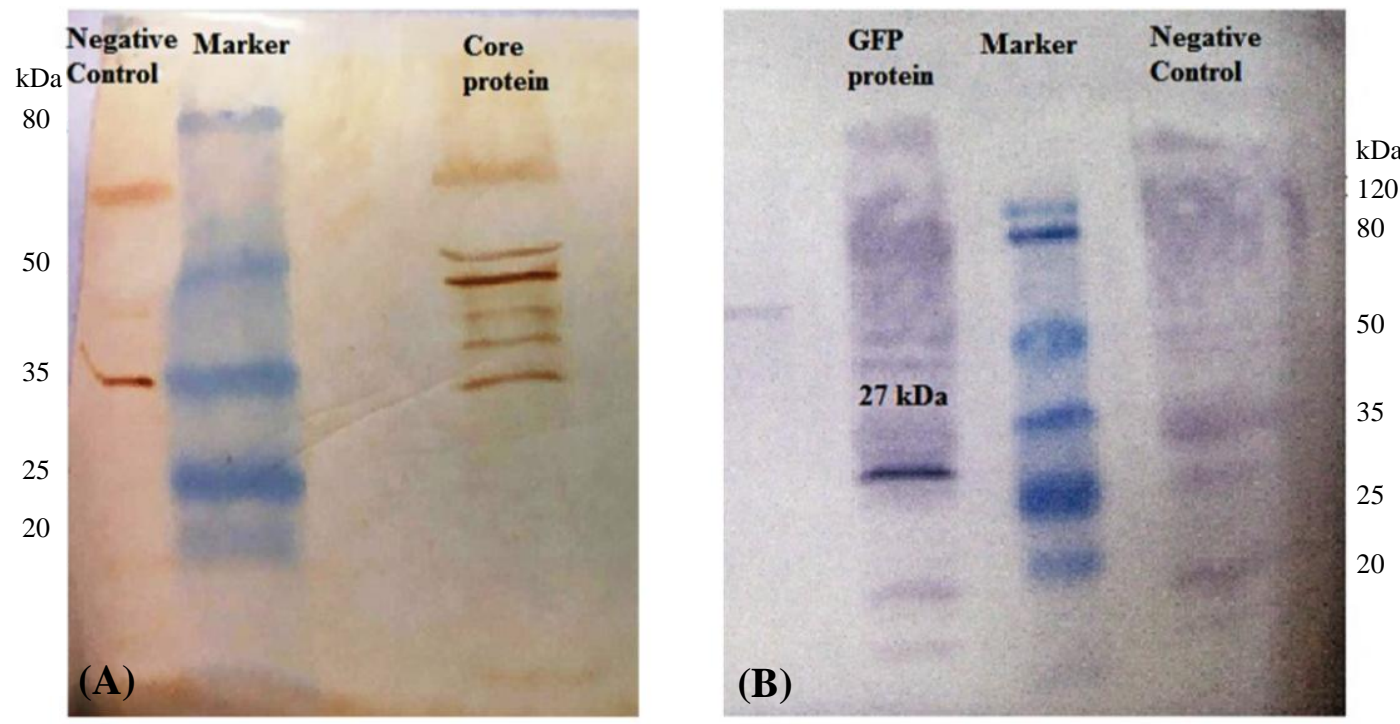

Fig. 6. Detection of expressed Core and GFP proteins by Western blot assay. (A) Core protein in transfected DCs. The right line of the marker shows Core protein, as a multi-band protein, with different molecular masses (37-55 kDa) in the transfected DCs, and the left line of marker shows the protein extracted from the untransfected DCs, as the negative control; (B) GFP protein in transfected DCs. The right line of marker is protein extract from the untransfected DCs, as negative control, and the left line of marker is GFP protein with the expected molecular mass $(27 \mathrm{kDa})$ in the DCs extract.

vaccine, it is essential to select an appropriate antigen and design mRNA with the correct structure composition. A common vector designed for the mRNA-based vaccine comprises of an ORF encoding the antigen of interest and optimized cis-acting flanking structures, with the 5' and 3' UTRs adjacent to the ORF, the terminal 5' 7-methylguanosine cap structure (cap), and the 3 ' poly (A). All of these elements help to enhance the antigen yield via maximizing translation speed and/or vector stability in transformed cells ${ }^{[40]}$.

Initiating a translation independent of the 5' cap has been well understood for viral mRNAs. For the first time in poliovirus, an alternative mechanism was found to start the translation, which occurs independent of the $5^{\prime}$ cap $^{[41]}$. Some viral mRNAs, such as picornaviral RNAs and HCV RNAs, often contain elements that directly promote translation initiation. One of these specific factors is a highly structured 5' UTRs, known as IRES, that can recruit ribosomes to the internal sequences of the mRNA lacking of $5^{\prime}$ cap structure. This mechanism is often exploited by mRNA expressions from the genome of the virus-infecting eukaryotic cells ${ }^{[41,42]}$. Typically, in some eukaryotic cells, the translation of mRNAs begins with the recruitment of the $43 \mathrm{~S}$ ribosomal complex to the $5^{\prime}$ cap of mRNAs. However, some transcriptions are translated independently of the 5' cap through unknown mechanisms ${ }^{[43]}$

Meyer et $a l^{[43]}$ have defined a unique translation initiation mechanism that does not require the 5' cap. Uncapped, luciferase-encoding mRNAs containing a modified $\beta$-globin $5^{\prime} \mathrm{UTR}$ with a single $\mathrm{m}^{6} \mathrm{~A}$ residue were used for in vitro translation assays. The results showed that the $\mathrm{m}^{6} \mathrm{~A}$ in the $5^{\prime}$ UTR can bind to the eukaryotic initiation factor 3 . The analysis of the transcriptome profiles showed that the translation of mRNAs from 5' UTR $\mathrm{m}^{6} \mathrm{~A}$ decreases with the deletion of $\mathrm{m}^{6} \mathrm{~A}$ methyl transferase ${ }^{[43]}$. Also, in the tobacco necrosis necrovirus, RNA is naturally translated, despite the lack of both 5' caps and poly (A) effectively, that is partly due to the presence of a component in 3' UTRs, which is called Barley yellow dwarf virus-like cap-independent translation element (BTE). Accordingly, in a study, the RNA encoding of luciferase reporter gene along with viral UTRs was used and uncovered a sequence downstream of the BTE that is required for translation without poly (A) in $v_{i v o}{ }^{[44]}$. Another study by Vivinus et al. ${ }^{[33]}$ have examined the mechanisms regulating the translation system for cellular proteins such as heat shock proteins that continue to be synthesized, despite the risk of translation conditions. They found that the 5' UTR of hsp70 mRNA contributes to the cap-independent translation without exhibiting typical features of IRES, but it does not behave as the viral IRES. In this study, 5' UTR of mouse hsp70 was used ${ }^{[33]}$. The effective translation of several mRNAs with the involvement of IRES in a number of cellular conditions is maintained as the cap-dependent protein synthesis is reduced. For 
instance, IRESs of the vascular endothelial growth factor and hypoxia-inducible factor-1a genes increase the translation of the corresponding mRNAs in hypoxic cells $^{[45]}$.

The analyses of studies followed the translation process without 5' cap and poly (A) in vitro have shown that the absence of $5^{\prime}$ cap and poly (A) compensates with the presence of IRES sequences in the upstream and downstream regions of the gene in the structure of the mRNA, and despite this defect, the translation process is done properly. Considering that most RNA viruses have the same structure and that such a structure is naturally responsive to the expression of viral proteins, it can be modeled to design and synthesize mRNA expressing variant antigens.

In this study, we considered the natural pattern of the RNA structure of the virus for the synthesis of the IVT-mRNA. Designed HCV RNA does not possess 5' cap and poly (A) tail. Instead, the IRES element (340 nucleotides) is located close to the 5' end of the viral genome ${ }^{[46]}$. The results of cell transfection demonstrated that despite the absence of the 5' cap and poly(A) tail, the structure of the IVT-mRNA was stable and was able to express the genes that it carried. The 5' cap plays an important role in the ribosomal recognition of messenger RNA when translated into a protein, and the $\operatorname{poly}(\mathrm{A})$ tail can also stimulate translation and cooperate with the cap structure ${ }^{[47]}$. It seems that the absence of the 5' cap and poly (A) tail in this structure is offseted by the presence of the IRES sequences that can directly recruit ribosomes under stress conditions and can bypass the need for a 5' cap, which is normally recognized by the translation initiation complex.

We designed a modified HCV antigen-coding mRNA, and computational evaluation showed its stability to create a stable secondary structure. Core antigen-coding sequence of $\mathrm{HCV}$ was successfully cloned into GE-30446-HCE vector, and its expression in the imoDCs transfected by transcribed IVT-mRNA confirmed the accuracy of designed mRNA. Considering the stability of the designed mRNA and its efficient expression in the effector cells such as moDCs, the synthesized IVT-mRNA can be used for further attempts in the development of RNA vaccine against $\mathrm{HCV}$.

\section{ACKNOWLEDGMENTS}

The authors would like to thank Drug Applied Research Center, Tabriz University of Medical Sciences, Tabriz, Iran for supporting this project and also appreciate our colleagues in the Department of Medical Biotechnology.
CONFLICT OF INTEREST. None declared.

\section{REFERENCES}

1. Kim CW, Chang KM. Hepatitis C virus: virology and life cycle. Clinical and molecular hepatology 2013; 19(1): 17-25.

2. Paintsil E, Binka M, Patel A, Lindenbach BD, Heimer R. Hepatitis $C$ virus maintains infectivity for weeks after drying on inanimate surfaces at room temperature: implications for risks of transmission. Thejournal of infectious diseases 2013; 209(8): 1205-1211.

3. Averhoff FM, Glass N, Holtzman D. Global burden of hepatitis $\mathrm{C}$ : considerations for healthcare providers in the United States. Clinical infectious diseases 2012; 55(suppl 1): S10-S15.

4. Mast EE, Alter MJ, Margolis HS. Strategies to prevent and control hepatitis B and C virus infections: a global perspective. Vaccine 1999; 17(13-14): 1730-1733.

5. Penin F, Dubuisson J, Rey FA, Moradpour D, Pawlotsky JM. Structural biology of hepatitis C virus. Hepatology 2004; 39(1): 5-19.

6. Shindo M, di Bisceglie AM, Hoofnagle JH. Long-term follow-up of patients with chronic hepatitis $\mathrm{C}$ treated with $\alpha$-interferon. Hepatology 1992; 15(6): 1013-1016.

7. Arends JE, Kracht PA, Hoepelman AI. Performance of hepatitis $\mathrm{C}$ virus (HCV) direct-acting antivirals in clinical trials and daily practice. Clinical microbiology and infection 2016; 22(10): 846-852.

8. Rosenthal ES, Graham CS. Price and affordability of direct-acting antiviral regimens for hepatitis $\mathrm{C}$ virus in the United States. Infectious agents and cancer 2016; 11(1): 24.

9. Sulkowski MS, Gardiner DF, Rodriguez-Torres M, Reddy KR, Hassanein T, Jacobson I, Lawitz E, Lok AS, Hinestrosa F, Thuluvath PJ, Schwartz H, Nelson DR. Daclatasvir plus sofosbuvir for previously treated or untreated chronic $\mathrm{HCV}$ infection. The new England journal of medicine 2014; 370(3): 211-221.

10. Ogholikhan S, Schwarz KB. Hepatitis vaccines. Vaccines (Basel) 2016; 4(1): 6.

11. Law LMJ, Landi A, Magee WC, Tyrrell DL, Houghton M. Progress towards a hepatitis $\mathrm{C}$ virus vaccine. Emerging microbes and infections 2013; 2(11): e79.

12. Li D, Huang $Z$, Zhong J. Hepatitis $C$ virus vaccine development: old challenges and new opportunities. National science review 2015; 2(3): 285-295.

13. Pardi N, Hogan MJ, Pelc RS, Muramatsu H, Andersen H, DeMaso CR, Dowd KA, Sutherland LL, Scearce RM, Parks R, Wagner W, Granados A, Greenhouse J, Walker M, Willis E, Yu JS, McGee CE, Sempowski GD, Mui BL, Tam YK, Huang YJ, Vanlandingham D, Holmes VM, Balachandran H, Sahu S, Lifton M, Higgs S, Hensley SE, Madden TD, Hope MJ, Kariko K, Santra S, Graham BS, Lewis MG, Pierson TC, Haynes BF, Weissman D. Zika virus protection by a single low-dose nucleoside-modified mRNA vaccination. Nature 2017; 543(7644): 248-251. 
14. Pascolo S. Messenger RNA-based vaccines. Expert opinion on biological therapy 2004; 4(8): 1285-1294.

15. Pascolo S. The messenger's great message for vaccination. Expert review of vaccines 2015; 14(2): 153156.

16. Probst J, Weide B, Scheel B, Pichler B, Hoerr I, Rammensee H, Pascolo S. Spontaneous cellular uptake of exogenous messenger RNA in vivo is nucleic acidspecific, saturable and ion dependent. Gene therapy 2007; 14(15): 1175-1180.

17. Sahin U, Karikó K, Türeci Ö. mRNA-based therapeutics-developing a new class of drugs. Nature reviews drug discovery 2014; 13(10): 759-780

18. McNamara MA, Nair SK, Holl EK. RNA-based vaccines in cancer immunotherapy. Journal of immunology research 2015; 2015: 794528.

19. Weiner DB. RNA-based vaccination: sending a strong message. Molecular therapy 2013; 21(3): 506-508.

20. Lutz J, Lazzaro S, Habbeddine M, Schmidt KE, Baumhof P, Mui BL, Tam YK, Madden TD, Hope MJ, Heidenreich R, Fotin-Mleczek M. Unmodified mRNA in LNPs constitutes a competitive technology for prophylactic vaccines. NPJ vaccines 2017; 2(1): 29.

21. Ahlquist $P$, French R, Janda M, Loesch-Fries LS. Multicomponent RNA plant virus infection derived from cloned viral cDNA. Proceedings of the national academy of sciences 1984; 81(22): 7066-7070.

22. Van Der Werf S, Bradley J, Wimmer E, Studier FW, Dunn JJ. Synthesis of infectious poliovirus RNA by purified T7 RNA polymerase. Proceedings of the national academy of sciences USA 1986; 83(8): 23302334.

23. Wolff JA, Malone RW, Williams P, Chong W, Acsadi G, Jani A, Felgner PL. Direct gene transfer into mouse muscle in vivo. Science 1990; 247(4949 Pt 1): 14651468.

24. Benteyn D, Heirman C, Bonehill A, Thielemans K, Breckpot K. mRNA-based dendritic cell vaccines. Expert review of vaccines 2015; 14(2): 161-176.

25. Morandi F, Chiesa S, Bocca P, Millo E, Salis A, Solari M, Pistoia V, Prigione I. Tumor mRNA-Transfected Dendritic Cells Stimulate the Generation of CTL That Recognize Neuroblastoma-Associated Antigens, Kill Tumor Cells: Immunotherapeutic Implications. Neoplasia 2006; 8(10): 833-842.

26. Kaczmarek JC, Kowalski PS, Anderson DG. Advances in the delivery of RNA therapeutics: from concept to clinical reality. Genome medicine 2017; 9: 60.

27. Loomis KH, Kirschman JL, Bhosle S, Bellamkonda RV, Santangelo PJ. Strategies for modulating innate immune activation and protein production of in vitro transcribed mRNAs. Journal of materials chemistry B 2016; 4(9): 1619-1632.

28. Bellaousov S, Reuter JS, Seetin MG, Mathews DH. RNAstructure: Web servers for RNA secondary structure prediction and analysis. Nucleic acids research 2013; 41(W1): W471-W474.

29. Spaggiari GM, Abdelrazik H, Becchetti F, Moretta L. MSCs inhibit monocyte-derived DC maturation and function by selectively interfering with the generation of immature DCs: central role of MSC-derived prostaglandin $\mathrm{E}_{2}$. Blood 2009; 113(26): 6576-6583.

30. Reuter JS, Mathews DH. RNAstructure: software for RNA secondary structure prediction and analysis. $B M C$ bioinformatics 2010; 11(1): 129.

31. Seffens W, Digby D. mRNAs have greater negative folding free energies than shuffled or codon choice randomized sequences. Nucleic acids research 1999; 27(7): 1578-1584.

32. Workman C, Krogh A. No evidence that mRNAs have lower folding free energies than random sequences with the same dinucleotide distribution. Nucleic acids research 1999; 27(24): 4816-4822.

33. Vivinus S, Baulande S, van Zanten M, Campbell F, Topley P, Ellis JH, Dessen P, Coste H. An element within the $5^{\prime}$ untranslated region of human Hsp70 mRNA which acts as a general enhancer of mRNA translation. The FEBS journal 2001; 268(7): 1908-1917.

34. Schlake T, Thess A, Fotin-Mleczek M, Kallen KJ. Developing mRNA-vaccine technologies. RNA biology 2012; 9(11): 1319-1330.

35. Soboleski MR, Oaks J, Halford WP. Green fluorescent protein is a quantitative reporter of gene expression in individual eukaryotic cells. The FASEB journal 2005; 19(3): 440-442.

36. Stretton S, Techkarnjanaruk S, McLennan AM, Goodman AE. Use of green fluorescent protein to tag and investigate gene expression in marine bacteria. Applied and environmental microbiology 1998; 64(7): 2554-2559.

37. Benteyn D, Anguille S, Van Lint S, Heirman C, Van Nuffel AM, Corthals J, Ochsenreither S, Waelput W, Van Beneden K, Breckpot K, Van Tendeloo V, Thielemans K, Bonehill A. Design of an optimized Wilms' tumor 1 (WT1) mRNA construct for enhanced WT1 expression and improved immunogenicity in vitro and in vivo. Molecular therapy nucleic acids 2013; 2: e134.

38. Petsch B, Schnee M, Vogel AB, Lange E, Hoffmann B, Voss D, Schlake T, Thess A, Kallen KJ, Stitz L, Kramps T. Protective efficacy of in vitro synthesized, specific mRNA vaccines against influenza A virus infection. Nature biotechnology 2012; 30(12): 1210-1216.

39. Kramps T, Probst J. Messenger RNA-based vaccines: progress, challenges, applications. Wiley interdisciplinary reviews: RNA 2013; 4(6): 737-749.

40. van der Velden AW, Voorma HO, Thomas AA. Vector design for optimal protein expression. Biotechniques 2001; 31(3): 572.

41. Pelletier J, Kaplan G, Racaniello V, Sonenberg N. Capindependent translation of poliovirus mRNA is conferred by sequence elements within the 5 ' noncoding region. Molecular and cellular biology 1988; 8(3): 1103-1112.

42. Jackson RJ, Howell MT, Kaminski A. The novel mechanism of initiation of picornavirus RNA translation. Trends in biochemical sciences 1990; 15(12): 477-483.

43. Meyer KD, Patil DP, Zhou J, Zinoviev A, Skabkin MA, Elemento O, Pestova TV, Qian SB, Jaffrey SR. 5' UTR 
m 6 A promotes cap-independent translation. Cell 2015; 163(4): 999-1010.

44. Shen R, Miller WA. Structures required for poly (A) tail-independent translation overlap with, but are distinct from, cap-independent translation and RNA replication signals at the $3^{\prime}$ end of Tobacco necrosis virus RNA. Virology 2007; 358(2): 448-458.

45. Stein I, Itin A, Einat P, Skaliter R, Grossman Z, Keshet E. Translation of vascular endothelial growth factor mRNA by internal ribosome entry: implications for translation under hypoxia. Molecular and cellular biology 1998; 18(6): 3112-3119.

46. Martínez-Salas E, Piñeiro D, Fernández N. Alternative mechanisms to initiate translation in eukaryotic mRNAs. Comparative and functional genomics 2012; 2012: Article ID 391546.

47. Gallie DR. The cap and poly (A) tail function synergistically to regulate mRNA translational efficiency. Genes and development 1991; 5(11): 21082116. 